\title{
Is an Anion Generating Face Mask the Ultimate Solution for Airborne Pollutants?
}

\author{
Madiha Khalid and Jeremy Madvin
}

\section{ABSTRACT}

\begin{abstract}
Airborne negatively charged ions have long been used for cleaning spaces by removing dust particles and stunting bacterial, fungal, and viral growth. Likewise, the ongoing pandemic has made the use of face masks inevitable. A novel concept has been established by integrating the benefits of negatively charged air ions with the advantages of conventional facemasks to tackle the current situation and offer additional advantages as well. The newly devised concept can be used by any individual who can wear a mask but will be especially useful for healthcare workers, infected individuals and those concerned about the possibility of inhaling harmful airborne particles. This new facemask concept may be crucial in reducing the risk of airborne diseases and is a far better choice in adequate protection as opposed to the standard single or multi layered cloth mask. This new mask concept is simple, cost effective, and appears to perform extraordinarily well. It requires no outside power source and is even washable and reusable. It is also remarkably comfortable and can be designed so that one size fits most adults.
\end{abstract}

Keywords: Anion Face Mask, Cloth mask, Negative ion face mask, TENG, Self-powered mask.
Submitted : March 26, 2021

Published : April 16, 2021

ISSN: $2593-8339$

DOI: $10.24018 /$ ejmed.2021.3.2.784

Dr. Madiha Khalid

Humdard University, Islamabad

Campus, Pakistan.

(e-mail: msmadihakhalid@gmail.com)

Jeremy Madvin*

MBA International Business

University of Redlands, United States.

(e-mail: Jeremymadvin@gmail.com)

*Corresponding Author

\section{INTRODUCTION}

In recent decades, emerging and re-emerging infections have become a health threat [1]. Various clinical diseases occur due to pathogens like bacteria, fungi, and viruses transmitted through the air [2]. Air transmission can be brought about by coughing, sneezing, talking or aerosol generation in any other way [3]. The World Health Organization (WHO) has attributed the transmission of airborne diseases to the spread of droplet nuclei that persist in the air for a long time and can cover a long distance to cause infections [4]. The world is facing a COVID-19 pandemic caused by the SARS-CoV-2 virus. The government imposed Lockdowns and social restrictions to minimize the spread of COVID-19, but it has a catastrophic impact on the economy. An infectious disease is controlled by knowledge of the way it is transmitted. Coronavirus is the cause of the COVID-19 pandemic, mainly transmitted through the respiratory tract [5], [6].

The social distance between people is widely referred to in non-pharmaceutical approaches to preventing diseases spread via the respiratory tract [7], [8]. Where safe distancing is not possible, personal protective equipment (PPE) is approved for self-defense. Perhaps the most critical piece of PPE are masks and respirators. They are a physical barrier to respiratory droplets and muco-salivary droplets, which may enter the nose and mouth from infected individuals [9], [10]. This plays a significant role when asymptomatic or presymptomatic infected persons may shed the virus [11], [12].
Several types of face masks are available in the market but vary in protection levels for users [13]. Masks can be either reusable or disposable. Reusable comprises commercial and homemade cloth masks; disposable masks include N95 respirators, KN95 respirators and surgical masks. They provide good protection from air contaminants, from pollen to chemical fumes to pathogenic agents. The mask's materials and engineering design determine its filtering capacity and safety level against pathogens and pollutants [14]. Factors like ease and breathability differ across various models in addition to filtering capacity. The majority of the masks have lower breathability, and after hours of wear, they can cause discomfort.

\section{ThE PURPose OF DEVEloping ANION GENERATING MASKS}

Masks can successfully aid in controlling the spread of infectious diseases [15], [16] and has thus become the new norm. The increasing demand for surgical masks and respirators has contributed to a global shortage of raw material and production. It has led many people creating their own masks, recycle masks or masks that provide less protection than desired and/or required. Therefore, researchers and industry stakeholders worked hard to solve the shortage and improve the safety offered by introducing new masking techniques. These efforts include:

1. the collection and construction of alternative materials with sufficient filter capacity, 
2. design of masks and respirators for improved safety, breathability, and level of comfort,

3. the development and engineering of antimicrobial, selfdisinfectant and even sensing capabilities, and

4. the exploration of other new technologies incorporated into customized masks.

It is clearly necessary to understand the fundamentals of mask technology to improve the performance of masks. Technologists focus on all the primary issues i.e.:

1. How masks protect from pathogens?

2. What type of mask materials are available in the market?

3. How masks work and how their performance compares with that of others?

4. How their shortcomings are to be improved?

5. How their performance can be increased?

6. What are some new features that can be incorporated into existing materials and models?

An ideal anion mask incorporates a design with a breathable substrate displaying small, precisely distanced anion emitters completely covering the nostril and mouth area to ensure the constant flow of anions but avoid repulsion, shown in Fig. 1. Each anion emitter is capable of continuous and consistent emission of anions without interruption. Each emitter is durable enough to withstand dozens, if not hundreds, of wash cycles. Each emitter operates without batteries or other external power sources. Each cubic centimeter of spotted material offers thousands of active anions at all times.

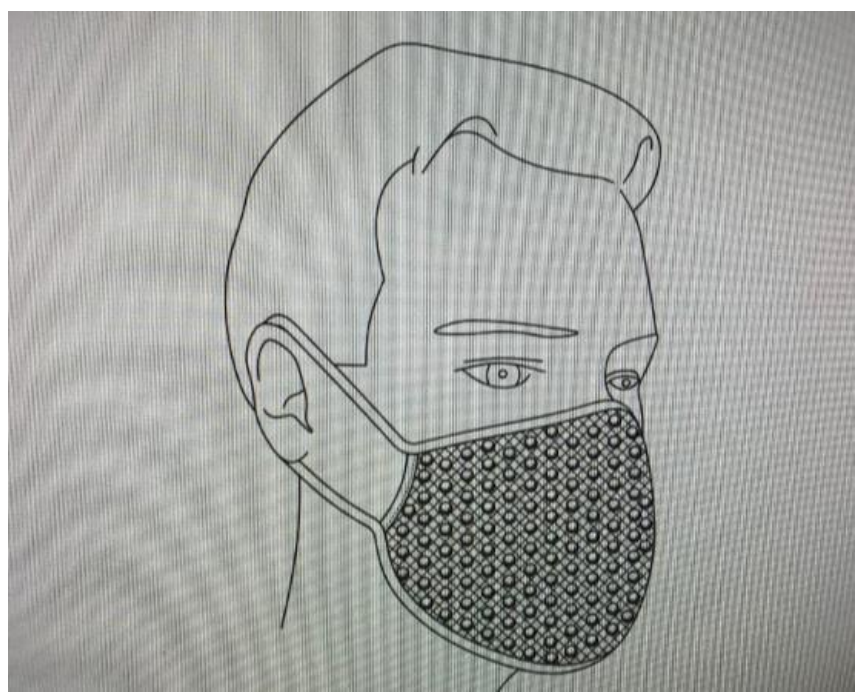

Fig. 1. Concept of Anion Mask.

\section{HOW ANION REMOVES HARMFUL AIRBORNE PARTICLES, VIRUSES AND BACTERIA?}

Literature has depicted that both negatively and positively charged ions can retard bacterial and fungal growth in solids, liquids, and air. The disturbance of electrical charges attacks the bacteria's stability, and negatively charged ions are more productive in this regard [17]. Ionization is a process whereby an atom loses or gains an electron. An ionizer emits negative ions into the environment. These negative ions attach with positive ions in the air like dust particles, fungal spores, pollen, and even airborne viruses and bacteria. Bonded molecules become heavier that leads them to drop or to settle down quicker on surfaces. It cleans the air and makes it free of unwanted particles but disinfects the surfaces manually or clean to remove bacteria and viruses. The ion generator's detergent qualities aid the breakdown of the surface proteins of allergens, bacteria, and viruses that help prevent airborne diseases. It boosts the body's defense against infections and harmful environmental factors. We use this principle in our face mask.

\section{HOW DOES THE ANION MASK WORK?}

The face mask aims to filter out the airborne particle or pathogens to prevent them from spreading infections in humans. Filtration systems for face masks must balance optimizing requirements. The pore size of filters must be small enough to catch particles but not too small enough to cause breathing difficulties; hence mask filters cannot fall below the average pore diameter. Anion masks work on three concepts to fulfill this optimization demand (Fig. 2):

1) Inertial Impact: Aerosol or dust particles usually larger than 1 micron have sufficient inertia to keep them from moving around the mask material and filter membranes and easily filter out.

2) Diffusion: Particles smaller than 1 micron that are not undergoing inertia. Diffuse and stick to the fibrous layers of the filter when rotating in a Brownian motion across the fiber's tortuous porous layer.

3) Electrostatic attraction: In this process, Electro charged polymer or resin fibers attract and capture both large and small oppositely charged particles.
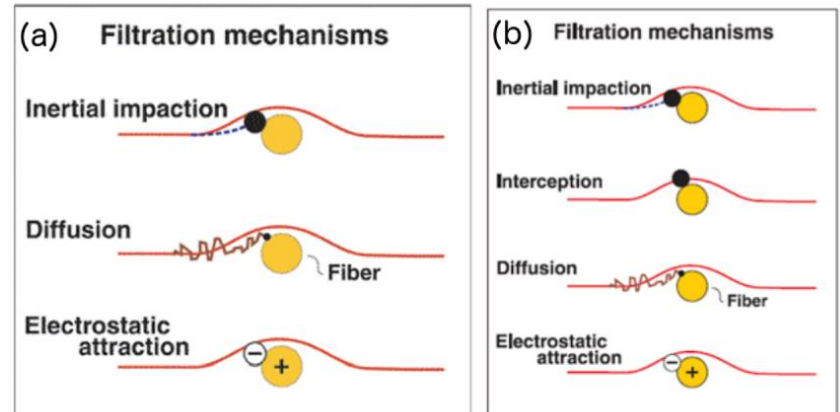

Fig. 2. The three filtration principles. (a) The National Academies Press report from 2006 details three stages (b) the CDC webpage lists four principles, but we have subsumed interception principle listed by CDC within inertial impaction principle.

Most face masks can seamlessly achieve the first and second principles. The third stage of electrostatic attraction is one of the design elements which distinguish the anion mask from other face masks.

\section{A. Electro charged Filtration}

In addition to mechanical filtration, anion masks use the principle of triboelectricity to improve electrostatic filtration and support electrostatic viral deactivation (Fig. 3). We know from everyday experience, especially in cold climates with low ambient humidity. When two fabrics rub against each other, they gain static electricity - a phenomenon commonly known as triboelectric charging.

Fabrics are woven from natural fibers like wool or cotton, which possess higher roughness, and even synthetic materials like Nylon gain static charge from rubbing. The idea of exploiting charged fabrics to aid infiltration goes back a few 
decades [18], and indeed, some early face mask designs incorporating electrocharged filtration did use wool or felt fibers. The anion face mask's inner layers continuously produce a tribe electric charge via the normal airflow of breath and speech. This charge uniquely provides additional defense while being harmless to the person wearing the mask.

Electrostatic attraction is used to trap big as well as small air particles. This approach considers electrically charged fibers or granules to absorb oppositely charged particles from the airstream in the filter [19]. For the nanometer scale, particles may slip between the openings in the filter fibers; electrostatic attraction is used to remove the small mass particles, and electrostatic filters can also serve on low velocity as respiratory velocity in the facemask [20].

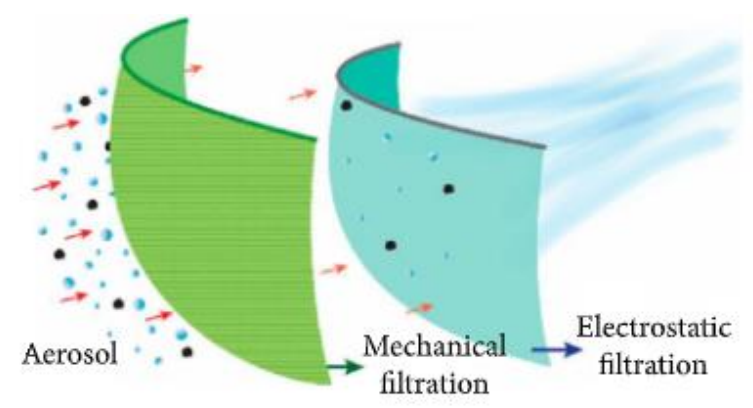

Fig. 3. Filtration mechanism in anion face Mask.

Triboelectricity generated in the mask serves as the electrical wall with advanced antimicrobial filtration provided by several layers of the cloth in the anion Mask. Research has demonstrated that triboelectricity and electroceutical fabrics may be used to destabilize viruses effectively. By Interaction with an electroceutical material for one minute, the viral particles' electrokinetic properties are significantly reduced. Triboelectricity dramatically helps to remove viruses without harming the mask user.

\section{B. Electret Membranes}

Unlike passive membranes, which collect particles primarily via their porous nature, electrostatic air filters can trap particles from a longer distance. The removal capacity can be retained under a constant airflow with a low-pressure drop without relying on a high density of tiny pores [21].

Charge dissipation can be solved using electret membranes fabricated using a tribocharging technique, resulting in improved filter stability and longer service life. For highefficiency particulate removal, a triboelectric nanogenerator (TENG) has recently been combined with nanofibrous air filters [22]. TENG is focused on triboelectrification, and the electrostatic induction effect generates high open-circuit Voltage.

A self-powered electrostatic adsorption face mask with a TENG operated by human respiration is created using this technology [22]. The removal ability is good under high humidity, and after washing cycles, there was no significant degradation. Tribo electric negative ion masks are thus highly efficient and reliable with a long service lifespan.

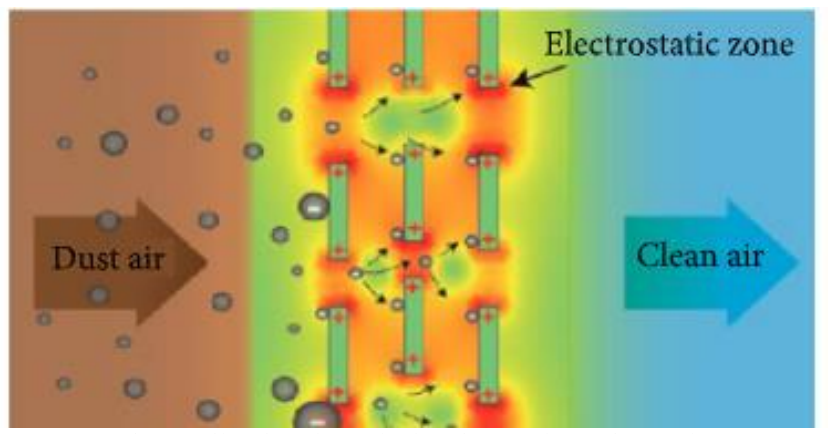

Fig. 4. Filtration through electric membrane in Mask.

\section{Convenience, Comfort, and Cleaning}

Since masks must be worn for long periods, the pain and discomfort, especially for children and the elderly, is exacerbated by current mask designs. It's normally a trade-off between comfort/breathability and filtration potential. Design and materials for anion face masks have been developed and invented that offering better breathability, comfort, $t$ and durability. Improving the material's softness/flexibility, ear loops, and weight can all help with the overall comfort of the mask.

\section{COMPARISON OF ANION GENERATING MASK}

\section{A. Comparison of Anion Generating Mask with Common Cloth Mask}

The following table (Table i) shows a fair comparison between the technically designed anion mask and singlelayered cloth mask [32].

TABLE I: COMPARISON BETWEEN SINGLE-LAYERED CLOTH MASK AND

\begin{tabular}{ccc} 
& ANION MASK & \\
\hline Properties & Cloth mask & Anion mask \\
\hline $\begin{array}{c}\text { Recommended for Anti-viral } \\
\text { effects }\end{array}$ & No & Yes \\
$\begin{array}{c}\text { Recommended for } \\
\text { Antibacterial effects }\end{array}$ & No & Yes \\
$\begin{array}{c}\text { Anti-asthma/allergic effects } \\
\text { Helps fight depression }\end{array}$ & Yes & Yes \\
Helps fight fatigue & No & Yes \\
Regulates heart and respiratory & No & Yes \\
rate & No & Yes \\
Risk of infection due to poor & Yes & No \\
filtration & Yes & Yes \\
Reusable & &
\end{tabular}

\section{B. Comparison of Anion Generating Mask with Plasma- Cluster Ions Technology}

Below (Table ii) is a brief comparison between plasmacluster and Anion-based technologies to ensure cleaning of the surrounding air and obtaining human health benefits [33], [34].

TABLE II: PLASMA-CLUSTER IONS VS. ANIONS

\begin{tabular}{ccc}
\multicolumn{2}{c}{ TABLE II: PLASMA-CLUSTER IONS VS. ANIONS } & \\
\hline Features & $\begin{array}{c}\text { Plasma-cluster } \\
\text { ions }\end{array}$ & Anions \\
\hline Anti-viral & Yes & Yes \\
Antibacterial & Yes & Yes \\
Anti-allergic & Yes & Yes \\
Uses both positive and negative & Yes & No, only \\
ions & No & anions \\
Depression management & Unknown & Yes \\
Regulates heartbeat, respiration, \\
and nervous system
\end{tabular}




\section{BENEFITS OF ANION BASED TECHNOLOGY}

Anion Air benefits can help solve numerous problems, as discussed here.

\section{A. Antibacterial Effect of Anions}

Possession of scientific knowledge has now changed the way we look at problems. Microorganisms can now be inactivated through desiccators, heaters, chemicals, ultraviolet radiation, and ionizers. The invention of carbon fiber-based ionizers has eased up air purification by a generation of unipolar ions. This technique has been tested against various bacteria like Escherichia coli and Staphylococcus epidermidis [23], [24].

The anions' antibacterial effect can be seen in the following graph [25].

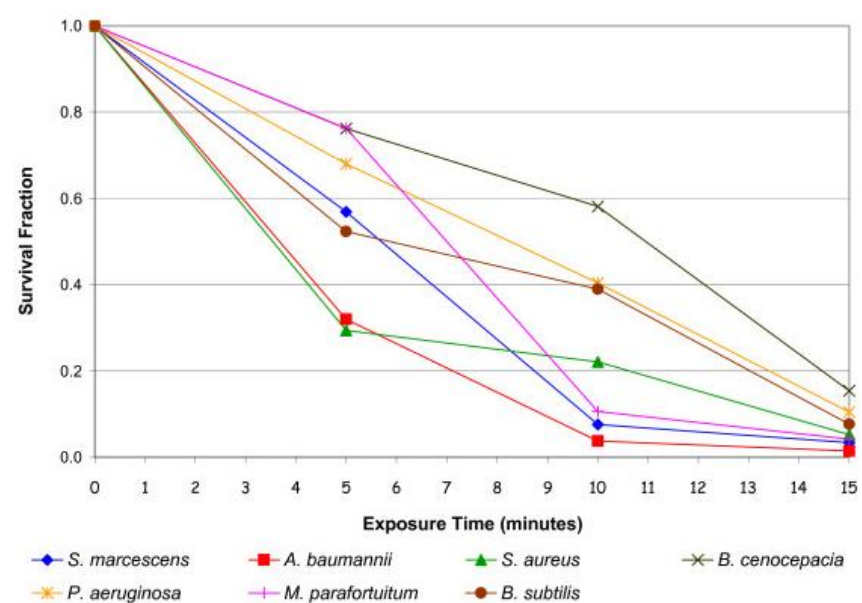

Fig. 5. Reduction in the viability of different bacterial species for the time after exposure to negative ions.

\section{B. Anti-viral Effect of Anions}

Anion-based technology has the potential to reduce the viral load from the air. In a study, Canine calicivirus and rotavirus aerosol particles were collected from the air using an ionizer. Viral particles of variable sizes can be inactivated in this way.

\section{Anti-allergic Effects of Anions}

Anions have been proved to have the ability to reduce allergenic effects generated by several allergens. A research study was conducted by using Japanese cedar pollen extract after being exposed to ions. As a result, the IgE from allergic patients was unable to bind the allergen. It was assumed that the allergic response could be minimized up to $80 \%$ by using this treatment [26].

\section{Anti-asthma Effects of Anions}

The administration of negative ion therapy has been explored in relation to asthma, as well. In children, shorter spasms were reported when they were treated with anions as opposed to conventional medicines. Abnormal and rapid breathing was minimized in cases with bronchopneumonia and bronchitis. The positive outcomes were reported within eight hours of therapy [27].

\section{E. Effects of Anions on the Nervous System, Heart Rate, and Respiratory Rate}

Negative ions have a reducing power and can maintain biological protection. They can be considered as the vitamins of air that have advantageous effects on human health. They assist the body in maintaining homeostasis. Moreover, the anions participate in the healing of damaged cells and contribute to cell vitality. Negatively charged ions can regulate nervous coordination, improve respiration and blood flow. They can also prevent, mitigate, or eliminate respiratory ailments [28].

\section{F. Anion Mediated Increase in Brain Serotonin Brings about Reduction in Stress and Fatigue}

Serotonin, mediated by 5-hydroxytryptophan, is produced by nerve cells if the number of cations entering the body is more than the anions. Serotonin, upon transportation to the tissues, hinders oxygen absorption into the bloodstream. The consequences of this incident may range from regular migraines, dizziness, fatigue, depression and even breathing issues. Hence there is a need to administer the negative ions through the nose, mouth, or skin. They can assist the conversion of 5-hydroxytryptophan to a harmless compound that can be excreted through urine. The putative anxiety disorder termed serotonin irritation syndrome can be reversed by using anions [29].

\section{G. Miscellaneous Positive Effects of Anions}

These negatively charged ions can actively promote the endocrine function and associated metabolism. They can also improve the immune system and enhance a person's working capacity. They can act as mood stabilizers and relaxers and help treat hypertension, heart diseases, and depression [30].

\section{H. Reduce Airborne Pollutants}

Tools that run on the principle of negative ion generation can potentially reduce viruses and bacteria, appropriately dispel the dust particles, pollutants, smoke, pollens, fungal spores, and airborne allergens from the air [32].

\section{CONCLUSION}

Due to the coronavirus pandemic, co-infections along with the prevailing coronavirus infections are commonly encountered. Healthcare governing bodies are trying their best to come up with a solution to tackle this problem. The WHO has requested individuals to follow the issued standard operating procedures and ensure personal hygiene. Safety can be maintained by observing physical distancing and using face masks. Depressed, quarantined, or self-isolated individuals can now obtain positive outcomes by using negative air ions. Face masks are a promising option to help at times when social distancing cannot be practiced. Masks created by anion technology show the potential to do wonders in elevating face masks' performance commonly found in the marketplace. 


\section{REFERENCES}

[1] S. Y. Wong and B. H. Tan, "Megatrends in infectious diseases: the next 10 to 15 years," Annals of the Academy of Medicine, Singapore, vol. 48, no. 6, pp. 188-194, 2019.

[2] Fernstrom, A. and M. Goldblatt, Aerobiology and its role in the transmission of infectious diseases. Journal of pathogens, 2013.

[3] Gammon, J. and J. Hunt, A review of isolation practices and procedures in healthcare settings. British Journal of Nursing, vol. 27, no. 3, pp. 137-140, 2018.

[4] Seto, W., Airborne transmission and precautions: facts and myths. Journal of Hospital Infection, vol. 89, no. 4, pp. 225-228, 2015.

[5] M. Xie and Q. Chen, "Insight into 2019 novel coronavirus -- an updated interim review and lessons from SARS-CoV and MERS-CoV," International Journal of Infectious Diseases, vol. 94, pp. 119-124, 2020.

[6] W.-j. Guan, Z.-y. Ni, Y. Hu et al., "Clinical characteristics of coronavirus disease 2019 in China," New England Journal of Medicine, vol. 382, no. 18, pp. 1708-1720, 2020.

[7] S. G. Benzell, A. Collis, and C. Nicolaides, "Rationing social contact during the COVID-19 pandemic: transmission risk and social benefits of U.S. locations," Proceedings of the National Academy of Sciences, vol. 117, no. 26, pp. 14642-14644, 2020.

[8] D. K. Chu, E. A. Akl, S. Duda et al., "Physical distancing, face masks, and eye protection to prevent person-to-person transmission of SARSCoV-2 and COVID-19: a systematic review and meta-analysis," The Lancet, vol. 395, no. 10242, pp. 1973-1987, 2020.

[9] J. W. Tang, T. J. Liebner, B. A. Craven, and G. S. Settles, "A schlieren optical study of the human cough with and without wearing masks for aerosol infection control," Journal of the Royal Society Interface, vol. 6, Supplement 6, pp. S727-S736, 2009.

[10] C. C. Leung, T. H. Lam, and K. K. Cheng, "Mass masking in the COVID-19 epidemic: people need guidance," The Lancet, vol. 395, no. 10228, p. 945, 2020.

[11] D. He, S. Zhao, Q. Lin et al., "The relative transmissibility of asymptomatic COVID-19 infections among close contacts," International Journal of Infectious Diseases, vol. 94, pp. 145-147, 2020.

[12] Z. Gao, Y. Xu, C. Sun et al., "A systematic review of asymptomatic infections with COVID-19," Journal of Microbiology, Immunology, and Infection, 2020.

[13] M. van der Sande, P. Teunis, and R. Sabel, "Professional and homemade face masks reduce exposure to respiratory infections among the general population," PLoS One, vol. 3, no. 7, article e2618, 2008.

[14] S. D. Rubbo and L. R. Abbott, "Filtration efficiency of surgical masks: a new method of evaluation," Australian and New Zealand Journal of Surgery, vol. 38, no. 1, pp. 80-83, 1968.

[15] N. H. L. Leung, D. K. W. Chu, E. Y. C. Shiu et al., "Respiratory virus shedding in exhaled breath and efficacy of face masks," Nature Medicine, vol. 26, no. 5, pp. 676-680, 2020.

[16] S. E. Eikenberry, M. Mancuso, E. Iboi et al., "To mask or not to mask: modeling the potential for face mask use by the general public to curtail the COVID-19 pandemic," Infectious Disease Modelling, vol. 5, pp. 293-308, 2020.

[17] Krueger, A.P. and E.J. Reed, Biological impact of small air ions. Science, vol. 4259, no. 193, pp. 1209-1213, 1976.

[18] Frederick, Edward R. "Some effects of electrostatic charges in fabric filtration." Journal of the Air Pollution Control Association vol. 24, no. 12, pp. 1164-1168, 1974.

[19] J. Bailar, D.S. Burke, L. Brosseau, H. Cohen, E. Gallagher, K. Gensheimber. Reusability of Facemasks during an Influenza Pandemic Institute of Medicine, National Academies Press, Washington [D.C.] (2006).

[20] A. Konda, A. Prakash, G.A. Moss, M. Schmoldt, G.D. Grant, S. Guha. Aerosol filtration efficiency of common fabrics used in respiratory cloth masks.ACS Nano (2020).

[21] P. P. Tsai, H. Schreuder-Gibson, and P. Gibson, "Different electrostatic methods for making electret filters," Journal of Electrostatics, vol. 54, no. 3-4, pp. 333-341, 2002.

[22] G. Liu, J. Nie, C. Han et al., "Self-powered electrostatic adsorption face mask based on a triboelectric nanogenerator," ACS Applied Materials \& Interfaces, vol. 10, no. 8, pp. 7126-7133, 2018.

[23] Kim, Y.S., et al., Application of air ions for bacterial de-colonization in air filters contaminated by aerosolized bacteria. Science of the total environment. vol. 409, no. 4, pp. 748-755, 2011.

[24] Lee, S.-G., et al., One-pass antibacterial efficacy of bipolar air ions against aerosolized Staphylococcus epidermidis in a duct flow. Journal of Aerosol Science. vol. 69, pp. 71-81, 2014.

[25] Fletcher, L.A., et al., Bactericidal action of positive and negative ions in air. BMC microbiology. vol. 7, no. 1, pp. 32, 2007.
[26] Kawamoto, S., et al., Decrease in the allergenicity of Japanese cedar pollen allergen by treatment with positive and negative cluster ions. International archives of allergy and immunology, 2006. 141(4): p. 313-321.

[27] Palti, Y., E. De Nour, and A. Abrahamov, The effect of atmospheric ions on the respiratory system of infants. Pediatrics, 1966. 38(3): p. 405-411.

[28] Jiajie, L., et al. Study on air anion concentration distribution in Beidaihe, China. in IOP Conference Series: Materials Science and Engineering. 2019. IOP Publishing.

[29] Giannini, A., B. Jones, and R. Loiselle, Reversibility of serotonin irritation syndrome with atmospheric anions. The Journal of clinical psychiatry, 1986. 47(3): p. 141.

[30] Goldstein, N., Reactive oxygen species as essential components of ambient air. Biochemistry (Moscow), 2002. 67(2): p. 161-170.

[31] English, J., The positive health benefits of negative ions. Nutri Rev, 2013. 28. MacIntyre, C.R., et al., A cluster randomised trial of cloth masks compared with medical masks in healthcare workers. BMJ open, 2015. 5(4): p. e006577.

[32] Ignelzi, R., Health Focus: Ways to Control Allergies. Network Journal, 1996: p. 15.

[33] Gefter, P. Biological aspects of clean-room ionization. in 2002 Electrical Overstress/Electrostatic Discharge Symposium. 2002. IEEE.

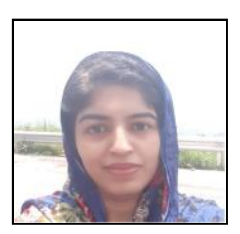

\section{Madiha Khalid}

Place of birth: Lahore.

Date of Birth: 11/9/1985.

M. Phil clinical Pharmacy..

Humdard University, Lahore

She is working as a lecturer in the University of

Lahore for about 6 years in the Pharmacy Department.

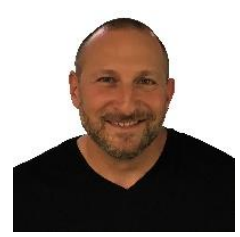

\section{Jeremy Madvin}

Place of Birth: Los Angeles, United States.

Bate of Birth: 11/22/1967.

MBA International Business.

University of Redlands, United States. 